\title{
Dural Arteriovenous Fistula Presenting with Purely Contralateral Ophthalmic Manifestations
}

\author{
Brandon J. Baartman ${ }^{\mathrm{a}}$ Andrew Bauer ${ }^{\mathrm{b}}$ Ferdinand Hui ${ }^{\mathrm{b}}$ Lisa Lystad ${ }^{\mathrm{a}}$ \\ Arun D. Singh ${ }^{\mathrm{a}}$ \\ a Department of Ophthalmic Oncology, Cole Eye Institute, and bepartment of Neuroradiology, Cleveland Clinic \\ Foundation, Cleveland, $\mathrm{OH}$, USA
}

\section{Keywords}

Dural arteriovenous fistula $\cdot$ Intracranial fistula .

Contralateral signs

\begin{abstract}
Purpose: To report a case of dural arteriovenous fistula presenting with purely contralateral ophthalmic manifestations. Design: Interventional case report. Results: A 70-yearold male presenting with right-sided proptosis, injection, and choroidal effusions with a completely quiet fellow eye was found to have a left-sided dural arteriovenous fistula. After recognition on cerebral angiography, successful endovascular embolization was achieved, along with complete resolution of the contralateral ocular signs. Conclusions: Entirely unilateral ocular signs suggestive of an intracranial arteriovenous fistula may herald the presence of a contralateral vascular lesion.

(c) 2016 S. Karger AG, Basel
\end{abstract}

\section{Introduction}

The cavernous sinus, as its name suggests, is a complex venous structure that may range from a single cavern to a septated, multilocular structure with pathways that may or may not communicate with the larger structure in which multiple compartments often exist [1]. Elevation of venous pressure anywhere along this system can transmit pressure to the cavernous sinus as well as the orbit, producing ophthalmic and neurologic signs dependent on the anatomy and flow of the lesion $[2,3]$.

A direct carotid-cavernous sinus fistula (CCF), defined as a direct communication between the intracavernous carotid artery and the surrounding cavernous sinus, produces a high-pressured system within the cavernous sinus itself. This high-flow vascular system produces the classic signs of orbital congestion including pulsatile proptosis, chemosis, and severe tortuous injection of the eye. In contrast, a dural-based arteriovenous fistula (DAVF) forms peripheral to the cavernous sinus involving meningeal branches of the internal or external carotid artery [2]. DAVF draining posteriorly into the superior and inferior petrosal sinuses may be largely asymptomatic, while those draining anteriorly can produce the classic ocular signs and symptoms resembling a direct CCF [2-4].

Abnormal communication between the arterial and venous circulation of the intracranial vasculature, whether a CCF or a DAVF, almost uniformly heralds the presence of an ipsilateral shunt. However, in a minority of documented CCF cases, cerebral angiography has revealed a fistula on the contralateral side [5-8].

\section{KARGER}

(c) 2016 S. Karger AG, Basel

E-Mail karger@karger.com

www.karger.com/oop
Arun D. Singh, MD

Department of Ophthalmic Oncology

Cole Eye Institute, Cleveland Clinic

2022 E 105th St, Cleveland, OH 44106 (USA)

E-Mail singha@ccf.org 
Fig. 1. Slit-lamp photograph of the right eye demonstrating dilated and tortuous episcleral vasculature. Also present but not represented here is the shallow nature of the anterior chamber. Posterior to the iris is an intraocular lens (a). Wide-field fundus photograph of the right eye demonstrating multilobulated choroidal effusions (b).
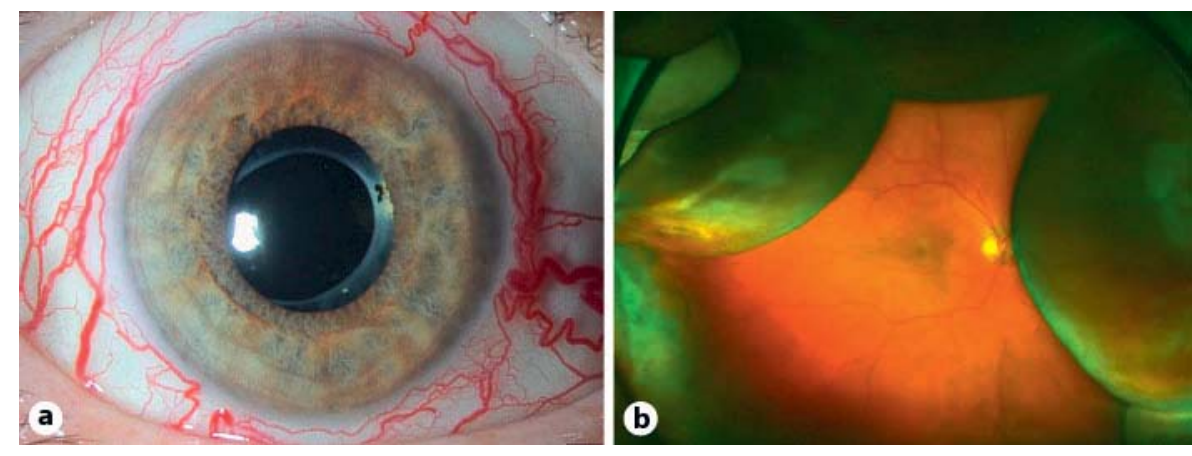

\section{Case Report}

A 70-year-old male presented to the Ophthalmic Oncology service with decreased vision, photophobia, and increasing redness in his right eye for 1 week. He was referred from another institution after being diagnosed with a retinal detachment. On examination, visual acuity in the right eye was 20/200 and the left eye was 20/25; there was no afferent pupillary defect. Intraocular pressures were normal in both eyes. External exam was notable for right-sided nonpulsatile proptosis of $4 \mathrm{~mm}$. The right eye had 360 degrees of prominent conjunctival and episcleral vessels and a shallow anterior chamber (Fig. 1a). Dilated fundus exam revealed multilobulated choroidal effusion and exudative retinal detachment (Fig. 1b). The left eye exam was unremarkable. Further history revealed remote head trauma in an industrial accident 16 years prior. Review of systems was notably negative for headache or other neurologic symptoms.

A right-sided arteriovenous fistula was suspected and CT imaging was obtained. However, this revealed what appeared to be a left-sided sphenoparietal DAVF (Fig. 2a, b). No abnormality in either superior ophthalmic vein was appreciated. Diagnostic cerebral angiography revealed a Borden class 3 DAVF fed from dural branches of the left middle meningeal artery and cavernous segment of the internal carotid artery and draining into the left sphenoparietal sinus and subsequently into the vein of Trolard which possessed a large venous varix, as well as through the cavernous sinus and into the contralateral superior ophthalmic vein (Fig. 2c, d). Selective injection of the inferior petrosal sinus revealed what was presumably partial opacification of the cavernous sinus with no drainage into the ophthalmic veins. The right internal and external carotid arteries were normal.

The patient then underwent endovascular embolization of the left sphenoparietal sinus via a retrograde venous approach from the superficial Sylvian vein resulting in complete obliteration of the fistula (Fig. 2e, f). On post-embolization day 1, the visual symptoms began to improve, with visual acuity as measured at bedside to be $20 / 30$. Postoperative course was complicated by transient aphasia, headache, and confusion with CT scan revealing a small left subdural hematoma, which was managed conservatively with complete recovery. The patient was seen by our Ocular Oncology service 1 month post-embolization with right eye visual acuity improving to 20/20 and normal intraocular pressure, and complete resolution of proptosis and the choroidal effusion (Fig. 3a, b).

Contralateral DAVF

\section{Discussion}

The majority of both CCF and DAVF present with only ipsilateral signs, with one study reporting a rate of $67 \%$ [3]. A minority of patients can be seen with bilateral signs attributable to drainage of the DAVF to bilateral anterior cavernous sinuses and orbital venous circulations. Contralateral ocular signs in the setting of a CCF has been described before, but the exact incidence is not known. The earliest documentation of CCF presenting with contralateral ocular signs was in an autopsy report by Dandy and Follis in 1941 [9], who suggested a thrombus at the entrance of the ophthalmic vein as the explanation for the transmittance of pressure and signs through the intercavernous sinuses to the contralateral orbit $[5,8]$. In 1966, Graham [5] discussed the case of a woman presenting with left-sided ocular signs in the presence of a right CCF which, left untreated by patient request, reversed in presentation with development of right-sided ophthalmoplegia and resolution of left-sided symptoms [5]. There has been suggestion in the neurosurgical literature that initial bilateral ocular signs may become more prominent on the contralateral side depending on the position of the fistula and its drainage patterns [6]. More recently, De Blasi and colleagues [7] reported a case and review of the literature revealing 18 cases of both traumatic and spontaneous CCF presenting with primarily contralateral ocular features. Martin et al. [8] also reported a case of CCF with symptoms exclusively contralateral to the arterial source.

All reported cases of contralateral signs were produced by CCF. In the case reported herein, the shunt was located in the sphenoparietal venous sinus without direct shunting into the cavernous sinus classifying it as DAVF. The drainage was not only through the vein of Trolard, but also into the network of veins along the cavernous sinus, across midline, and into the contralateral superior

Ocul Oncol Pathol 2017;3:106-109 
Fig. 2. Axial computed tomography with contrast shows dilated veins (arrow) in the area of the left sphenoid wing and paraclinoid area (a). A large venous varix (arrow) is also seen on the vein of Trolard coursing toward the sagittal sinus (b). Anteroposterior (c) and lateral (d) digital subtraction angiography with injection in the left internal carotid artery. From the internal carotid injection, the dural arteriovenous fistula is seen fed by small dural branches of the lateral cavernous sinus (white arrow) draining directly into the sphenoparietal sinus. This, in turn, drains to the superficial Sylvian vein and vein of Trolard which has a large venous varix (black arrow). Angiography with injection in the left inferior petrosal sinus shows no direct involvement of the cavernous sinus with the fistula. Postembolization with injection from the left common carotid artery (e, anteroposterior; $\mathbf{f}$, lateral). The coil mass is seen in the sphenoparietal sinus (arrow). There is no further filling of the fistula from the internal or external carotid arteries and there is no early venous drainage.

Fig. 3. Normal external (a) and fundus appearance (b) 1 month after embolization of the dural arteriovenous fistula.
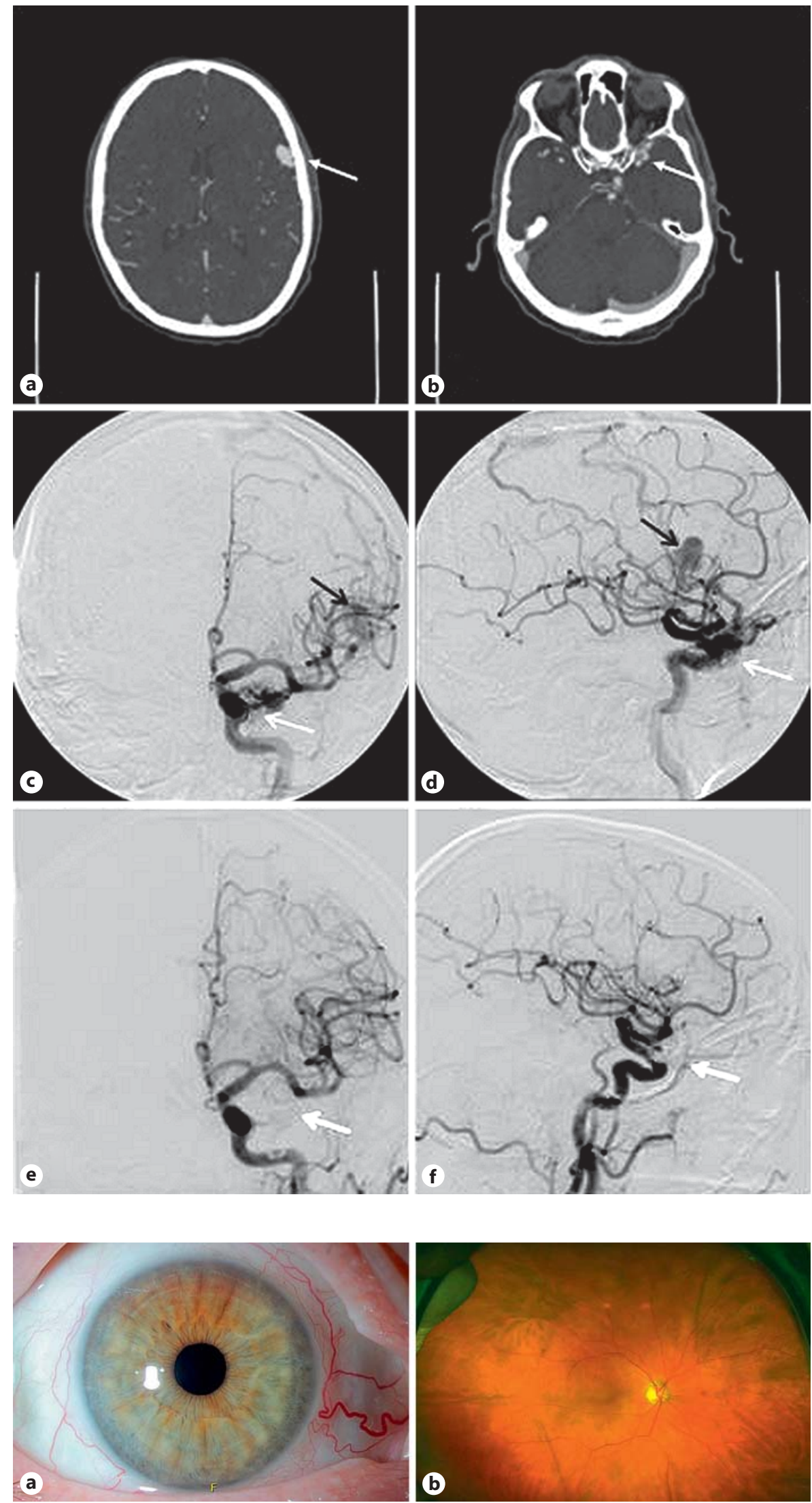
ophthalmic vein. This implies a channel parallel and separate from the cavernous sinus draining the sella turcica as demonstrated on injection of the inferior petrosal sinuses. Presence of this additional channel is the proposed explanation for the contralateral signs exhibited by our patient.

Coiling of the shunt pouch, without treatment of the cavernous sinus, resulted in complete obliteration of the shunt, and resolution of contralateral orbital-venous arterialization and congestion. Ophthalmologists should remain suspicious of intracranial pathology in the setting of such unusual presentations and be aware that culprit lesions may indeed be contralateral to the side of symptoms.

\section{Statement of Ethics}

The study complied with the guidelines for human studies and animal welfare regulations. The subject gave informed consent, and the study protocol was approved by the institute's committee on human research.

\section{Disclosure Statement}

The authors have no conflicts of interest to declare.

\section{References}

1 Harris FS, Rhoton AL Jr: Anatomy of the cavernous sinus: a microsurgical study. J Neurosurg 1976;45:169-180.

2 Miller NR: Dural carotid-cavernous fistulas: epidemiology, clinical presentation, and management. Neurosurg Clin N Am 2012;23: 179-192.

3 Stiebel-Kalish H, Setton A, Nimii Y, Kalish Y, Hartman J, Bar-On RH, Berenstein A, Kupersmith MJ: Cavernous sinus dural arteriovenous malformations: patterns of venous drainage are related to clinical signs and symptoms. Ophthalmology 2002;109:16851691.
4 De Keizer RJW: Carotid-cavernous and orbital arteriovenous fistulas: ocular features, diagnostic and hemodynamic considerations in relation to visual impairment and morbidity. Orbit 2003;22:121-142.

5 Graham MV: Arteriovenous shunt in the cavernous sinus with contralateral ocular signs. Br J Ophthalmol 1966;50:599-602.

6 Hawkins TD: Case report: traumatic carotidcavernous fistula with contralateral proptosis. Clin Radiol 1986;37:509-512.
7 De Blasi R, D'Urso PI, Colamaria A, Occhiogrosso G, Ciapetta P: Spontaneous carotidcavernous fistula supplied by the contralateral menigohypohyseal trunk: case report and literature review. J Neurosurg Sci 2010;54: $45-48$.

8 Martin TJ, Troost BT, Ginsberg LE, Tegeler $\mathrm{CH}$, Weaver RG: Left dural to right cavernous sinus fistula. J Neuroophthalmol 1995;15:3135.

9 Dandy WE, Follis RH: On the pathology of carotid-cavernous aneurysms (pulsating exophthalmos). Am J Ophthalmol 1941;24: 365-385. 